A C A S E

OF COMPLETE

\title{
INVERSION OF THE UTERUS,
}

\section{OF NEARLY TWELVE YEARS' DURATION, SUCCESSFULLY TREATED.}

BY

W. TYLER SMITH, M.D.,

PHYSICIAN-ACCOUCHEUR TO, AND LECTURER ON MIDWIFERY AT, ST. MARY's HOSPITAL.

Received Dec. 31st, 1857.-Read April 13th, 1858.

The tenth and thirty-fifth volumes of the 'MedicoChirurgical Transactions' contain the histories of two cases of inversion of the uterus occurring after parturition; the first by Mr. John Windsor, of Manchester, and the second by Mr. Gregory Forbes. In one of these cases extirpation was effected, and the patient recovered; in the other, palliative measures were resorted to, and the patient died of exhaustion eighteen months from the date of delivery. These cases are scarcely more interesting or remarkable than that which is the subject of the present paper, and as the latter illustrates a new method of treating this formidable affection, I have ventured to bring it before the notice of the Society.

Hitherto, inversion of the uterus has generally been 
treated either by styptics and astringents, or the inverted organ has been removed by ligature or excision. The instances in which reinversion has been accomplished have been few in number, and chiefly limited to cases of recent origin. Mr. Forbes, who gives a very excellent account of current opinions on this subject, states that "with regard to the possibility of reducing the inverted organ, it may be admitted, on the testimony of the most competent observers, that unless this desirable end can be effected within a few hours after the accident has occurred, there is little or no hope of its being afterwards accomplished." He observes that, in a few rare and exceptional cases, reduction has been effected at a later period, and of the cases he himself collected, one had been reposited fifteen months after the inversion had occurred. In still more rare instances, it has been found that the accident has led to ro great hæmorrhage or other serious symptom; or that spontaneous reinversion has taken place. But the result, in the vast majority of cases, where the inverted uterus remains unrelieved, is undoubtedly a fatal one. Mr. Forbes refers to fourteen cases in which death followed at periods varying from eight months to five years after the occurrence of inversion. As regards the serious operation of extirpating the inverted organ, the paper of Mr. Forbes also contains some important statistical information. Of twenty-six cases treated by ligature, the operation was successful in twenty-one. In five cases it was unsuccessful, and of these three died. In the other two the ligature had to be removed soon after its application. Of two cases treated by excision, one recovered, and one died. In eight cases treated by the ligature and excision combined, five were successful, and three were unsuccessful. Thus, of thirty-four cases of extirpation, twenty-seven recovered, and seven died; but probably in this, as in other formidable operations, the results appear more favorable than they really are, from the non-publication of unsuccessful cases. To show the difficulty of diagnosis in inversion of the uterus, it may be mentioned that in no less than nine of the preceding cases, the uterus was mistaken 
for polypus. With these remarks, I proceed to relate the case which fell under my own observation.

CASE.-Mrs. J-, æt. 18, was delivered of a first child on the 6th of October, 1845. Immediately after the completion of labour her medical attendant left the house, having received a summons to another midwifery case, at a considerable distance in the country. Mrs. J - lost a very large quantity of blood after his departure, and was said to have remained insensible for ten or twelve days. At the end of this time there was no swelling externally, and no suspicion of inversion was excited. She continued, however, to lose blood, which at first was considered to be the lochial discharge in unusul quantity, but none of the means used were sufficient to arrest the hæmorrhage. The flooding continued to a greater or less extent for nearly twelve years. During this long time she was never for a single day free from sanguineous discharge. Before her pregnancy, the catamenia had been regular and moderate. From the time of her labour, the loss of blood generally increased at the monthly dates, and remained profuse for upwards of a fortnight, after which the discharge would become paler for about ten days, until the date of the next monthly return; but it never ceased entirely. The extent of the hæmorrhage may be gathered from the fact that during the fortnight of excessive loss, from fifteen to twenty thick napkins would become saturated every twenty-four hours. When this state of things had continued for some time, she was examined, and the existence of a tumour in the vagina was detected. The opinions of the many medical men who saw her were divided as to whether the case was one of inverted uterus, or of polypus descending after labour, as sometimes occurs. The existence of this doubt prevented any operative means for the removal of the tumour, beyond abortive attempts to replace the uterus, by those who considered it a case of inversion. Astringents given internally, and used externally, with every other means that could be devised, failed of affording any relief. 
In July, 1856, Mrs. J- was sent to me from Port Madoc, North Wales, where she resided, by Mr. Griffith, under whose care she had been for a short time only, and from that gentleman, and the patient herself, I learnt the foregoing particulars of her case. When I first saw her, she was in a state of extreme anæmia. The skin was exceedingly pale and wax-like; the conjunctivæ were without any visible trace of blood-vessels, the tongue and gums were ash-coloured, as was also the mucous membrane of the vulva and vagina. She was not particularly thin, but flabby, and the lower extremities and the body generally, were odematous. The pulse was scarcely perceptible at the wrist. When she was at rest the sounds of the heart were regular but weak, and loud venous murmurs were audible in the neck. Her appetite was habitually bad, and a sense of nausea, often proceeding to vomiting, was very distressing. She suffered severely from cephalalgia. Dimness of vision, sometimes amounting to blindness, tinnitus aurium, palpitation increased to a most alarming extent by slight exertion, and great breathlessness. On many occasions she had fainted and remained insensible for several hours. She had also been subject to convulsions of an epileptic character. Sleeplessness was a very marked feature in her case. She rarely slept soundly, and on falling off to sleep was constantly roused by a feeling of faintness and dissolution. This was increased if she lay on her left side. The sleeplessness and breathlessness mentioned are, I believe, constant attendants upon excessive and long-continued loss of blood. It appears as though, in such extreme cases, respiration could not go on without the assistance of volition, and as if the system could scarcely afford that amount of cerebral congestion necessary to sleep.

The effects of the constant hæmorrhage on the secretions were very remarkable. She described herself as never having been in a perspiration since the loss of blood commenced. The action of the skin seemed to be entirely suspended. The bowels were obstinately constipated. She made very little water, and constantly went twenty-four 
hours or more, without passing any urine at all. It appeared as if the loss of blood took the place, to a considerable extent, of the several secretions, and as if in this way the system had become, to some extent, accustomed to the drain from the uterus. Every succeeding year she had, however, become sensibly weaker, and the fits of fainting and insensibility had grown more frequent and lasting.

On making a digital examination, a pyriform tumour could be felt within the vagina, hanging from the os uteri. It was difficult to make out by the finger whether the os and cervix were inverted, or whether the tumour hung as a polypus from the interior of the uterus. The os uteri was small and rigid, and the neck of the tumour was of corresponding size. No amount of sudden pressure could have forced the mass of the tumour upwards, without rupturing the os and cervix uteri. A probe, or the uterine sound, could not be passed more than half an inch within the os, showing that no uterine cavity existed. The structure of the tumour did not appear harder or more elastic than in some cases of firm fibrous polypi, in which degeneration has not commenced. I have felt some cases of polypi which could hardly have been distinguished by the touch alone, from the tumour in the present case. The size was somewhat larger than the natural size of the unimpregnated uterus. The sensation communicated to the finger was very much like that of a mass of India-rubber, softened at the surface. Examined by the speculum, and with a fine probe, nothing like the openings of the Fallopian tubes could be detected. The mucous membrane, like the mucous covering of polypi, was seen to be shreddy and ulcerated in patches. Some parts of the tumour were smeared with pus, and at others blood freely exuded from its surface. The mass was comparatively insensible to the touch, and the prick of a needle or the pinch of a pair of forceps gave little or no pain ; but, on one occasion, when a ligature was passed round the neck of the tumour, and held tightly for a short time, most acute suffering was produced.

Mr. Griffith, when he sent the patient to me, considered 
the case to be one of inversion, and after several examinations, I arrived at the same conclusion, though some experienced accoucheurs to whom I showed the case, among whom I may mention the late Dr. Ashwell, inclined to the opinion that it was polypus. Acting, however, on the belief that the uterus was inverted, I resolved to attempt its reduction by continuous pressure. As the tumour was not painful, and as the circulation and respiration were feeble and distressed, I did not consider it prudent to use chloroform, or to effect any sudden replacement, for which chloroform is so well adapted in comparatively recent cases, but depended on the effects of moderate and sustained pressure, with the view of dilating or developing the os and cervix uteri, so as to admit of the return of the inverted organ. With this intention I passed the right hand into the vagina night and morning for several days, and endeavoured by squeezing and moulding the uterus with the fingers for about ten minutes at a time, to press the tumour upwards. At first no impression could be made in this way, but after repeated trials, I found the cervix uteri yield a little, and the tumour could be sunk slightly in the os. On each occasion, after removing the hand, I passed one of $M$. Gariel's large air-pessaries into the vagina, and inflated it to as great extent as the patient could bear. By this means a very considerable force was constantly exerted upon the tumour. The air-pessary was worn day and night, with few exceptions; or when it was removed on account of pain, or for the purpose of relieving the bladder and bowels. From the time of the first introduction of the pessary, the hæmorrhage ceased entirely, and the tumour became somewhat less in size. On each succeeding day, it could be passed a little higher within the os uteri. After more than a week of these proceedings, the patient felt a good deal of pain through the whole of one night, and in the morning, when an examination was made, it was discovered that complete reversion had taken place.

The os uteri was now found to be considerably dilated, and readily admitted the end of the finger. The sound could be passed fully three inches into the uterine cavity. There 
was a considerable amount of muco-purulent discharge, and on examining with the speculum, the os was found to be abraded and patulous, but otherwise healthy. A small-sized air-pessary was worn for a few days, and the recumbent position preserved; but no further hæmorrhage, nor any attempt at inversion occurred. The patient began to eat and sleep well, and in the course of a fortnight returned to Carnarvonshire, greatly improved in health. The leucorrhœa soon ceased, by the use of simple remedies, under the care of Mr. Griffith. During the greater part of the manipulations which were necessary, I was assisted by Dr. Vernon, at that time the resident obstetric officer at St. Mary's Hospital.

Since the return of Mrs. J - to her home, I have frequently heard of her through Mr. Griffith, who describes her as having lost the appearance of anæmia, and become stout and healthy. Menstruation has continued regularly, and in moderate quantity.

The important questions for consideration are, first, whether the foregoing is a rare and exceptional case of reinversion, such as those which have been recorded from time to, time; and, secondly, whether the means by which the replacement was effected, are likely to prove useful in other cases, and to supply a principle of treatment in the cure of inversion. In the former point of view, the case would be of little or no value, except as an obstetric curiosity, though I believe there is no other case on record in which an inversion of such long standing has been reduced; if the latter question should be answered in the affirmative, it of course becomes of considerable importance. I proceed to state my reasons for believing that the use of air or fluid pressure may be made of general application in the reduction of all chronic cases of inverted uterus.

An idea has always been entertained that the uterus which has been inverted for a considerable time is in a hope. less state of immobility. As soon as the accident has happened, the powerful contractions of the os and cervix 
upon the inverted portion, and the great size of the latter, render its return a matter of great difficulty. This difficulty is further increased, when the involution of the uterus or its return to the unimpregnated size, has taken place. Instead of mere contraction, it is now the structural condition of the organ which opposes its reinversion. The comparatively bulky body and fundus, of almost cartilaginous hardness, cannot be made to pass through the contracted and rigid os uteri, by any mechanical force short of the rupture or laceration of the parts concerned. This is undoubtedly true, when the inverted uterus is treated as a mechanical displacement, and sought to be remedied by simply mechanical means.

But there is in the uterus a principle of growth, diminution, and alteration of form, under appropriate stimuli, or their withdrawal, surpassing that of any other part of the body, which I believe may be used in the reduction of inversion, and upon which I depended in the foregoing case. The presence of the ovum, excites the enormous development of the gravid uterus. Its return to the unimpregnated condition after the removal of this stimulus by delivery, is equally remarkable. The organ enlarges in the same manner, but to a smaller extent, under the stimulus of a contained polypus, or of a fibrous tumour within its walls. In cases of ovarian disease, also, that side of the uterus in proximity to the tumour, often enlarges to a considerable extent. In polypus, when the tumour has attained a large size, the virgin os and cervix will become developed to such an extent as to permit the passage of the polypus. Under the influence of continuous irritation, the virgin uterus has even been known to invert itself completely, a much more difficult matter, it might be supposed, than the reposition of the uterus when inverted after parturition. We already make use of the principle referred to, therapeutically, in promoting the growth of the imperfectly developed uterus, when this condition is combined with sterility and amenorrhœa; and in dilating or developing a constricted cervix, in dysmenorrhœa, or in enlarging the cervical canal 
when we desire a polypus to pass, by the use of spongetents, and other means. In the same way, from à priori reasoning, and from what I observed in the preceding case, I believe that the necessary development may be gradually imparted to the os and cervix, in cases of inversion, so as certainly to admit the return of the organ. In the case under consideration, nothing could have been more rigid and unpromising than the os and cervix uteri; but in obedience to the force and stimulus exerted, chiefly by the air-pessary, the fundus and body of the uterus was converted into a wedge or tent, which by constant pressure dilated and developed the narrow ring which had so long fixed the uterus in its inverted position. At the same time, the pressure diminished the size of the body and fundus, and in this way promoted the re-inversion, and the arrest of the hæmorrhage. I should entertain little doubt of the possibility of replacing the uterus in any case of inversion, by similar means, and so far from the uterus being considered one of the most difficult and obstinate, it ought to be held as one of the most manageable organs of the body, in any matter relating to growth or form. The plan of treatment by air or fluid pressure must at least deserve a trial in all future cases, when we consider that the alternatives are, death from loss of blood, or the extirpation of the uterus, with its attendant risks.

Besides the employment of the air-pessary in the vagina, to effect reinversion of the uterus in the case described, I have used it with good effect for the arrest of hæmorrhage, in cases of menorrhagia resisting ordinary remedies, and to keep the uterus replaced in cases of retroversion. I have no doubt the same means would prove serviceable, in cases of flooding from fibrous tumour of the uterus; or from polypus, when the state of the os uteri does not admit of operation. It offers a better kind of tampon than any hitherto used, in cases of flooding during abortion, and in placenta prævia, before the dilatation of the os uteri. I have also used the air-pessary before the time of labour, in a case of high deformity of the pelvis from mollities ossium, with con- 
siderable effect, in separating the contracted bones. The case I refer to, I saw with Dr. S. W. J. Merriman, and before the use of the pessary, the tuberosities of the ischia were so close as scarcely to admit the passage of one finger. After the separation of the bones, delivery was effected by turning. Professor Carl Braun, of Vienna, Kiwisch, and others, have likewise used the air-pessary with great success as a means of inducing premature labour, and of hastening delivery, by promoting the dilatation of the os uteri, in puerpural convulsions, the new method being termed colpeurysis.

Postscript.-October 19th, 1858. A few days after the reading of the preceding paper, I received a note from $\mathrm{Mr}$. Griffith, to whom I was indebted for this interesting case, informing me of the pregnancy of Mrs. J-S Since then she has been delivered of a living child under his care. Some amount of flooding occurred after labour, but no tendency to inversion was manifested. 